\title{
LONG-ACTING PREDNISOLONE IN THE CONTROL OF LEPRA REACTION
}

\author{
By S. G. BrownE, M.D., F.R.C.P., F.R.C.S., D.T.M. \\ and \\ L. M. Hogerzeil, Med. Drs. (Leyden) \\ Leprosy Service Research Unit, Uzuakoli, Eastern Nigeria
}

The value of the corticosteroids in controlling severe lepra reaction is generally admitted. Not only do they in appropriate dosage alleviate or abolish its distressing clinical manifestations, but under their cover specific anti-leprosy therapy may be continued, unaltered or suitably modified.

The limitations and drawbacks of corticosteroid therapy are likewise recognized, and the restricted indications for the use of these expensive products have become better defined.

There is a class of patients suffering from advanced lepromatous leprosy, with generalized infiltration and perhaps widespread nodulation, highly positive bacteriologically at all sites, who develop during the course of treatment (or spontaneously) severe and persistent lepra reaction that is amenable only to corticosteroid therapy over long periods. When their corticosteroid requirements are relatively stable and not subject to fluctuation from day to day, such patients have been treated for weeks or months with "maintenance" doses of cortisone (JOPLING and CochRANE, 1957) or prednisolone.

To overcome the many practical disadvantages of prolonged divided-dose oral prednisolone therapy, with its possible side-effects (especially gastro-intestinal disturbances due to high local concentration of the product, and amyloid degeneration of the liver), a trial was instituted at Uzuakoli with a long-acting prednisolone given at weekly intervals by intramuscular injection. The drug was Deltacortril brand of prednisolone (Pfizer), a sterile aqueous suspension of prednisolone acetate $(25 \mathrm{mg}$. per $\mathrm{ml}$.) which is released at a regular rate from a depot injection. The actual duration of effective prednisolone concentration following an intramuscular injection has been deduced from clinical and from urinary corticosteroid assay findings (WEST, 1958), and depends apparently on both the need of the individual and the amount injected; such factors as local vascularity and local reaction are also of some importance; the average duration of such concentration is seven days.

\section{Choice of patients}

Twelve adult lepromatous patients participated in the trial: they had all had severe lepra reaction for many months, for which they 
had received a variety of treatments. Only oral prednisolone given in three divided doses daily had proved effective in controlling the reaction and permitting continuation of anti-leprosy therapy. They represented all such patients then under treatment at Uzuakoli whose corticosteroid requirements had for some time been reasonably stable. Each patient had hitherto received regularly that dose of prednisolone just sufficient to abolish erythema nodosum leprosum and to relieve nerve pain so as to permit sleep without sedatives. Any reduction in the amount of prednisolone administered would result within 36 hours in a recrudescence or exacerbation of the symptoms.

\section{Dose}

The dose of intramuscular prednisolone was calculated as seven times the total daily requirements of oral prednisolone. Oral treatment was continued for 24 hours after the injection had been given to allow for an equivalent time-lag in release of the active substance from the depot injection. Since the individual requirements were known, it was not necessary to ascertain by trial and error the initial effective minimal maintenance dose.

The twelve patients received on the average 15 weekly intramuscular injections of the product.

\section{Results}

In all patients, the calculated doses of the intramuscular prednisolone were found to be approximately correct: the symptoms of lepra reaction were controlled as with oral prednisolone, but for a duration of about seven days after a single injection. All patients were at first ambulant, but three of them were admitted to bed-rest in hospital for a time during the course of treatment-a proportion similar to that expected in such a group.

Five of the patients definitely preferred the injected to the oral prednisolone, volunteering that the symptoms (especially nerve pain and general malaise) were more effectively controlled. A rather sudden and dramatic wave of contentment and well-being was noted by four patients about twelve hours after each injection.

\section{Modification of dosage}

While weekly intramuscular injections cannot be as flexible as daily divided doses of the comparable product given orally, indications for altering the dose were provided by the clinical evidence of the duration of effective concentration of prednisolone: $v i z$., if symptoms tended to reappear before the end of the seventh day after the injection, the day of the next injection could be anticipated and the dose slightly increased; conversely, if the symptoms were fully controlled for the whole seven days, then the next injection could be postponed or the dose slightly decreased. 
The erythrocyte sedimentation rate was of no value in indicating the degree of clinical response to injected prednisolone.

Side-effects were virtually absent.

No abscess formed at the sites of the injections; none of the patients suffered from any infection.

No evidence of fluid retention, such as moon-face or oedema, was seen. Some patients lost weight, and some gained slightly: diet and exercise seemed to account for any change that occurred.

The patients all followed their normal diets; no supplementary potassium salts were given. No change in appetite was reported.

While a general sense of well-being was frequently noticed by the patients themselves, no excessive euphoria or psychic disturbance or insomnia occurred.

There was no case of hirsutism, or reappearance of lost eyebrows.

No spontaneous fracture occurred.

No evidence of toxic action on liver, kidneys or pancreas was found.

\section{Variation in corticosteroid requirements}

One patient, stable for some months, but before then inherently liable to violent fluctuations in his reactional state, suddenly developed a severe exacerbation requiring temporarily much higher doses of corticosteroids to control the widespread erythema nodosum and general symptoms. The injected prednisolone was supplemented without difficulty by oral prednisolone given in divided doses for as long as the augmented requirements persisted.

\section{Resumption of oral corticosteroid therapy}

At the conclusion of the trial, it was possible without difficulty to resume oral prednisolone therapy at the appropriate dose level. Since equivalent oral therapy was resumed when intramuscular therapy was discontinued, no withdrawal symptoms were encountered.

\section{Continuation of anti-leprosy therapy}

Before oral prednisolone had been given to these patients, many had been unable to tolerate dapsone (DDS) or thiambutosine (DPT) in adequate dosage. Under oral prednisolone cover, all but three were able to take adequate doses of one or other drug; afterwards, during intramuscular prednisolone therapy, those three were given standard doses of thiambutosine without ill effects.

\section{Summary and Conclusion}

Twelve lepromatous patients, all suffering from prolonged lepra reaction (relatively stable in its degree of severity) controllable only by corticosteroid oral therapy, were given prednisolone acetate 
(Pfizer) in aqueous suspension by intramuscular injection at weekly intervals for 15 weeks.

The symptoms were adequately controlled, and anti-leprosy therapy was continued, or resumed.

Side-effects of prolonged corticosteroid therapy in appropriate dosage adjusted to individual requirements, were negligible.

There is thus a definite place for a long-acting injectable prednisolene in the control of severe long-standing lepra reaction in lepromatous patients whose corticosteroid requirements are relatively stable. Under such cover, it is possible to continue anti-leprosy therapy.

It is hoped that, with increased production, and commercial competition, the price of this valuable class of product may be so reduced as to bring it within the reach of all who need it.

\section{Acknowledgments}

Our thanks are due to Messrs. Pfizer for generous supplies of their brand (Deltacortril) of prednisolone acetate prepared for intramuscular injection, and to Dr. S. E. Onwu, M.v.o., O.B.E., Director of Medical Services and Permanent Secretary, Ministry of Health, Eastern Nigeria, for permission to publish.

\section{References}

Jopling, W. H. and Cochrane, R. G. (1957 Leprosy Review, 38, 5.

WEST, H. F. (1958) Ann. rheum. Dis., 17, 273. 